\title{
Pedestrian Protection Using the Integration of V2V and the Pedestrian Automatic Emergency Braking System
}

\author{
Bo Tang, Stanley Chien, Senior Member, IEEE, Zhi Huang, Student Member, IEEE, and Yaobin Chen \\ Senior Member, IEEE
}

\begin{abstract}
Vehicle to Vehicle (V2V) communication systems enable vehicles to communicate with each other and use the shared information to make safety related decisions. However, the safety improvement of the current V2V systems only benefits V2V-enabled objects in the V2V network. The Pedestrian Automatic Emergency Braking System (PAEB) can utilize onboard sensors to detect pedestrians and make safety related actions so it benefits the individual vehicle and the pedestrians detected by its PAEB. To further improve pedestrian safety, the idea for integrating the capabilities of V2V and PAEB (V2V-PAEB) has been proposed, which allows the information of pedestrians detected by onboard sensors of a vehicle to be shared in the V2V network. A V2V-PAEB enabled vehicle uses not only its onboard sensors, but also received V2V messages from others to detect potential collisions with pedestrians and make better safety related decisions. In this paper, a Matlab/Simulink based simulation model of V2V-PAEB system is presented for demonstrating the proper architecture and information processing processes, and for providing the quick start of developing a better simulation model of V2V-PAEB. The proposed model has also been tested in PreScan simulation environment.
\end{abstract}

Index Terms - Intelligent vehicles, radar detection, real-time systems, vehicle detection, vehicle safety

\section{INTRODUCTION}

Due to the fast advancement of wireless communication technology and computation speed, Vehicle-to-Vehicle $(\mathrm{V} 2 \mathrm{~V})$ communication becomes practical and being implemented in many research studies. The information exchange through $\mathrm{V} 2 \mathrm{~V}$ enabled vehicles to make better decisions in driving control and safety [1]. Meanwhile, Pedestrian Automatic Emergency Braking (PAEB) systems use various types of onboard sensors (such as radar, mono/stereo camera, infrared etc.) to detect the potential collision with pedestrians. The PAEB alerts the driver if there is an imminent collision and supports collision avoidance by applying the brake automatically if the driver does not take braking action [2]. Due to the range limitations of PAEB sensors and speed limitations of sensory data processing, PAEB systems often cannot detect or do not have sufficient time to respond to a potential crash. To improve the pedestrian safety, the idea of integrating the complimentary

All authors are with the Indiana University-Purdue University Indianapolis. $723 \mathrm{~W}$. Michigan St., Indianapolis, IN 46202, USA. Contact e-mail:schien@iupui.edu. capabilities of $\mathrm{V} 2 \mathrm{~V}$ and PAEB (V2V-PAEB) together to allow the information of pedestrians sensed by PAEB of one vehicle to be shared in the V2V network is proposed. The information broadcasted through V2V network can be used by the PAEB of other vehicles.

A set of 96 scenarios that the pedestrian safety can benefit from the proposed V2V-PAEB feature has been identified [3]. Based on the V2V-PAEB features discussed in [3], this paper standardizes the V2V-PAEB functions and features, where it also provides some convincible demonstrations. These scenarios proved the potential use of the V2V-PAEB and could be used for evaluating V2V-PAEB systems. These simulated scenarios can also illustrate the feasibility in the real world. However, successful V2V-PAEB actions are highly depending on the number of vehicles with the V2V-PAEB hardware.

Based on the state-of-the-art V2V, V2P (Vehicle-to-Pedestrian) communication standards [1], this paper concentrates on the detailed feasibility and experimental analysis. This paper presents a Matlab/Simulink based computer simulation architecture specially designed for the further development of V2V-PAEB system and its simulation model. This simulation architecture is organized according to the information processing steps and the problems need to be solved in a V2V-PAEB system. It is called architecture since it captures all necessary parts of a V2V-PAEB for it to be functional and allows the flexibility of future improvement of any individual subsystem. Goals for developing this simulation model architecture include: (1) present the problems and challenges in developing the V2V-PAEB system; (2) decompose the problem into a set of well-defined sub-problems; (3) present the architecture and the information processing flow for solving all sub-problems; (4) provide a simple implementation of the simulation model to demonstrate the usefulness of the model for studying the V2V-PAEB integration problems and (5) providing a foundation for quick verification of new V2V-PAEB algorithms. With the predefined architecture and function blocks described in this paper, new V2V-PAEB information processing algorithms can be quickly evaluated. The V2V-PAEB simulation model has been tested in the PreScan environment. Some pedestrian safety related V2V-PAEB experiments described in [3] were implemented by incorporating V2V-PAEB algorithms to demonstrate the usefulness of this model. 


\section{Description of V2V-PAEB SimUlation MOdel}

The V2V-PAEB simulation model connected with its required supporting upstream/downstream models in a vehicle model is shown in Figure 1. The block in the center is the V2V-PAEB simulation model, and blocks on both sides are peripheral upstream/downstream models. The V2V-PAEB model uses information from upstream models to detect potential collisions with pedestrians and make proper safety decisions. Then these decisions are sent to downstream models to trigger proper actions to avoid or mitigate the potential collisions. Various types and numbers of sensors can be used in V2V-PAEB system. The output of the sensors models provides the position, velocity, and acceleration of the detected objects. Sensors can be added to or removed from the V2V-PAEB simulation model. Current implementation of the V2V-PAEB simulation model supports two basic sensor models (radar and camera).

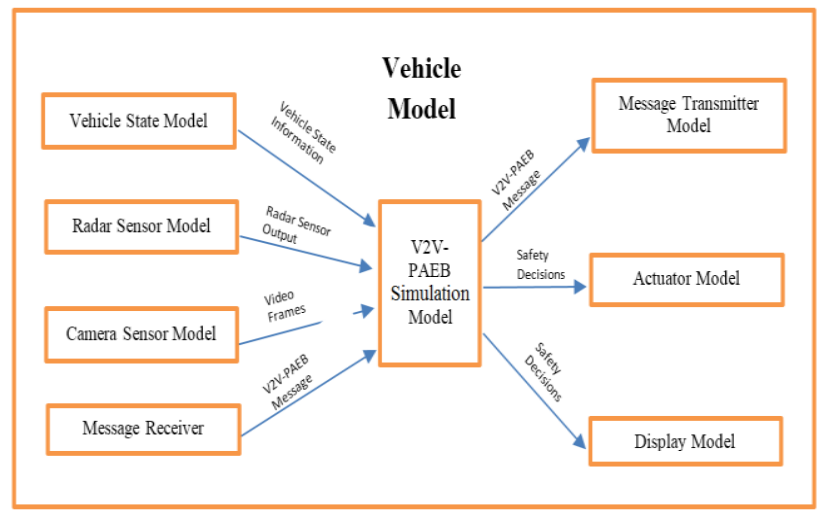

Figure 1. V2V-PAEB model in a vehicle model.

The V2V-PAEB simulation model provides the ability of message passing (transmitting and receiving) with well-defined message protocol. V2V-PAEB Message is a subtype of $\mathrm{V} 2 \mathrm{~V}$ communication message. It contains the information of pedestrians detected by their V2V-PAEB systems. TABLE 1 shows the required input of V2V-PAEB simulation model. The format of the V2V-PAEB Message is defined in TABLE 2. The detailed information of the output data of the V2V-PAEB simulation model is shown in TABLE 3.

TABLE 1. THE INPUT DATA OF V2V-PAEB MODEL

\begin{tabular}{|l|l|}
\hline Item & Description \\
\hline $\mathrm{X}, \mathrm{Y}, \mathrm{Z}[\mathrm{m}]$ & vehicle coordinates in global coordinate system \\
\hline $\begin{array}{l}\text { GPS } \\
{[\mathrm{deg} / \mathrm{min} / \mathrm{sec}]}\end{array}$ & $\begin{array}{l}\text { The GPS Latitude, Longitude, and Altitude of the } \\
\text { host vehicle }\end{array}$ \\
\hline $\begin{array}{l}\text { Rotation about } \\
\mathrm{X}, \mathrm{Y}, \mathrm{Z}[\mathrm{deg}]\end{array}$ & $\begin{array}{l}\mathrm{X}, \mathrm{Y}, \text { and } \mathrm{Z} \text {-rotations of the vehicle in global } \\
\text { coordinate system. }\end{array}$ \\
\hline Yaw Rate [deg/s] & The Yaw (Turning) rate of the vehicle. \\
\hline Velocity [m/s] & The moving velocity of the vehicle. \\
\hline $\begin{array}{l}\text { Heading } \\
\text { Direction }[\mathrm{deg}]\end{array}$ & $\begin{array}{l}\text { The moving direction of the vehicle. North is } 00 \\
\text { (range between } 0-360 \text { in clockwise direction). }\end{array}$ \\
\hline $\begin{array}{l}\text { Acceleration } \\
{[\mathrm{m} / \mathrm{s} 2]}\end{array}$ & The acceleration of vehicle. \\
\hline $\begin{array}{l}\text { Throttle State } \\
{[\%]}\end{array}$ & Throttle state of vehicle. Range between $0-100$. \\
\hline
\end{tabular}

\begin{tabular}{|l|l|}
\hline Brake State [\%] & Brake state of vehicle. Range between 0-100. \\
\hline $\begin{array}{l}\text { Steering Angle } \\
\text { [deg] }\end{array}$ & The steering Angle of vehicle. \\
\hline Radar model & $\begin{array}{l}\text { The radar sensor can be configured with a number of } \\
\text { beams with different angle coverages. This Beam ID } \\
\text { indicates which beam is active in the current } \\
\text { simulation time step. }\end{array}$ \\
\hline Beam ID [-] & $\begin{array}{l}\text { The distance between the radar sensor and the } \\
\text { detected objects. }\end{array}$ \\
\hline Range (R) [m] & $\begin{array}{l}\text { Velocity of target point, relative to the sensor, along } \\
\text { the line of-sight between sensor and target. }\end{array}$ \\
\hline $\begin{array}{l}\text { Doppler } \\
\text { Velocity [m/s] }\end{array}$ \\
\hline Theta $\Theta$ [deg] & Azimuth angle in the sensor coordinate system \\
\hline Phi Ф[deg] & Elevation angle in the sensor coordinate system \\
\hline Target ID [-] & Numerical ID of the detected target. \\
\hline $\begin{array}{l}\text { Energy Loss } \\
\text { [dB] }\end{array}$ & Ratio received power / transmitted power. \\
\hline Alpha $\alpha$ [deg] & Azimuthal incidence sensor angle on the target. \\
\hline Beta $\beta[$ deg] & Elevation incidence sensor angle on the target. \\
\hline TIS Data [-] & Communication bus signal contains sensor's output. \\
\hline $\begin{array}{l}\text { Doppler } \\
\text { Velocity X/Y/Z } \\
\text { [m/s] }\end{array}$ & $\begin{array}{l}\text { Velocity of target point decomposed into X, Y, Z of } \\
\text { the sensor's coordinate system. }\end{array}$ \\
\hline $\begin{array}{l}\text { Target Type ID } \\
\text { Radar may identify the type of detected objects. The } \\
\text { Type ID specifies the detected object type. }\end{array}$ \\
\hline $\begin{array}{l}\text { Vamera Model } \\
\text { rate and } \\
\text { format }\end{array}$ & $\begin{array}{l}\text { The camera sensor model should be able to generate } \\
\text { video frames at a proper frame rate. }\end{array}$ \\
\hline Message Receiver Model \\
\hline $\begin{array}{l}\text { Message } \\
\text { Table } 2 \text { shows the message format. }\end{array}$ \\
\hline
\end{tabular}

TABLE 2. THE FORMATION OF V2V-PAEB MESSAGE

\begin{tabular}{|l|l|}
\hline Item & Description \\
\hline Header & $\begin{array}{l}\text { The destination of the message if unicast is } \\
\text { supported. }\end{array}$ \\
\hline Destination & The sender of this message. \\
\hline Source & Type of message (e.g., V2V-PAEB type). \\
\hline Type of message & $\begin{array}{l}\text { The subtype of the message. In case for } \\
\text { expanding this message in the future. }\end{array}$ \\
\hline Subtype of message & The priority of the message. \\
\hline Priority of message & The event time stamp of the message. \\
\hline Event time & Total packages of the message. \\
\hline Total packs & The pack id of current message. \\
\hline Packet id & The length of the payload of this message. \\
\hline Length of payload & Host vehicle's unique ID in the V2V network. \\
\hline Payload & To indicate the type of the host vehicle \\
\hline Vehicle ID & Host vehicle's color. \\
\hline Vehicle type & $\begin{array}{l}\text { Host vehicle's latitude, longitude and altitude } \\
\text { [deg]. }\end{array}$ \\
\hline Vehicle color & Host vehicle's moving velocity [m/s]. \\
\hline $\begin{array}{l}\text { Vehicle GPS } \\
\text { location }\end{array}$ & $\begin{array}{l}\text { Host vehicle's heading direction. North is } 0 \\
\text { degree range between 0-360o clockwise. }\end{array}$ \\
\hline Vehicle speed & The acceleration of the host vehicle. \\
\hline Vehicle heading & The accuracy of host vehicle's GPS device. \\
\hline Vehicle acceleration & $\begin{array}{l}\text { To indicate how many pedestrians' } \\
\text { information are included in this message }\end{array}$ \\
\hline $\begin{array}{l}\text { Vehicle GPS } \\
\text { accuracy }\end{array}$ & The ID of the first pedestrian \\
\hline $\begin{array}{l}\text { Number of } \\
\text { pedestrians }\end{array}$ & The confidence level of this Ped information \\
\hline Pedestrian ID & The color of the first pedestrian \\
\hline Confidence & The latitude, longitude and altitude of the first \\
\hline Pedestrian size & \\
\hline Pedestrian color & Pedestrian GPS
\end{tabular}




\begin{tabular}{|l|l|}
\hline location & pedestrian \\
\hline Pedestrian velocity & The velocity of the first pedestrian $[\mathrm{m} / \mathrm{s}]$ \\
\hline Pedestrian heading & first pedestrian's heading direction $[\mathrm{deg}]$ \\
\hline Ped. acceleration & The acceleration of the first pedestrian[m/s2] \\
\hline Pedestrian ID & $\begin{array}{l}\text { The ID of the second pedestrian. Second } \\
\text { pedestrian's information stars here. }\end{array}$ \\
\hline$\ldots \ldots$ & $\ldots \ldots$ \\
\hline$\ldots \ldots$ & $\begin{array}{l}\ldots \ldots . \\
\text { rest of detected objects. }\end{array}$ \\
\hline
\end{tabular}

TABLE 3. INFORMATION OF THE OUTPUT DATA OF THE V2V-PAEB SiMULATION MODEL

\begin{tabular}{|c|c|}
\hline $\begin{array}{l}\text { Object Type } \\
\text { ID }\end{array}$ & Description \\
\hline $\begin{array}{l}\text { Pedestrian } \\
\text { Detection } \\
\text { Flag } \\
{[\mathrm{Y} / \mathrm{N}]}\end{array}$ & $\begin{array}{l}\text { This flag is used to indicate whether any pedestrians } \\
\text { have been detected. If so, this parameter will be set to } \\
\text { Y. Otherwise, it will be set to N. }\end{array}$ \\
\hline $\begin{array}{l}\text { Driver } \\
\text { Warning } \\
\text { Flag }[\mathrm{Y} / \mathrm{N}]\end{array}$ & $\begin{array}{l}\text { This flag is used to indicate if the driver warning } \\
\text { should be triggered. If this parameter is set to Y, then } \\
\text { a driver warning will be triggered immediately. } \\
\text { Otherwise, if this parameter is set to N, then the } \\
\text { vehicle will do nothing. }\end{array}$ \\
\hline $\begin{array}{l}\text { Automatic } \\
\text { Braking Flag } \\
{[\mathrm{Y} / \mathrm{N}]}\end{array}$ & $\begin{array}{l}\text { This flag is used to indicate if the automatic braking } \\
\text { should be started. If it is set to Y, then the automatic } \\
\text { braking will be started immediately. Otherwise, if } \\
\text { this parameter is set to N, then the vehicle will do } \\
\text { nothing. }\end{array}$ \\
\hline $\begin{array}{l}\text { Brake } \\
\text { Pressure } \\
\text { [bar] }\end{array}$ & $\begin{array}{l}\text { This value is used to control the deceleration of } \\
\text { the vehicle when the automatic braking is started. } \\
\text { Once the Automatic Braking Flag is set to Y, then the } \\
\text { Brake Pressure should be assigned a value between } \\
\text { zero and the Max Braking pressure. Otherwise, if the } \\
\text { Automatic Braking Flag is set to N, then this } \\
\text { parameter should be zero. }\end{array}$ \\
\hline $\begin{array}{l}\text { Automatic } \\
\text { Steering Flag } \\
{[\mathrm{Y} / \mathrm{N}]}\end{array}$ & $\begin{array}{l}\text { This flag is used to indicate if the automatic steering } \\
\text { control should be started. If it is set to } \mathrm{Y} \text {, then the } \\
\text { automatic steering will be started immediately. } \\
\text { Otherwise, if this parameter is set to } \mathrm{N} \text {, then the } \\
\text { vehicle will do nothing. }\end{array}$ \\
\hline $\begin{array}{l}\text { Automatic } \\
\text { Steering } \\
\text { Angle } \\
{[\text { deg] }}\end{array}$ & $\begin{array}{l}\text { This value is used to specify the steering wheel } \\
\text { status. A positive value means turning right, and } \\
\text { minus value means turning left. Once the Automatic } \\
\text { Steering Flag is set to } Y \text {, then this parameter should } \\
\text { be assigned with a meaningful value. } \\
\text { Otherwise, it should be zero. }\end{array}$ \\
\hline $\begin{array}{l}\text { Time To } \\
\text { Collision [s] }\end{array}$ & $\begin{array}{l}\text { Time to Collision represents for how much time } \\
\text { left before the collision occurs. If many pedestrians } \\
\text { are detected at the same time, and each one has its } \\
\text { TTC. Then this parameter will be set to the smallest } \\
\text { of them. }\end{array}$ \\
\hline $\begin{array}{l}\text { V2V-PAEB } \\
\text { Message } \\
\text { [array] }\end{array}$ & $\begin{array}{l}\text { The V2V-PAEB Message that contains the } \\
\text { pedestrians that detected by PAEB system on } \\
\text { vehicle. } \\
\text { Vehicles use this message to share the pedestrian } \\
\text { information through V2V communication. }\end{array}$ \\
\hline
\end{tabular}

There are two types of output data generated by the V2V-PAEB simulation model: the V2V-PAEB Message and safety decisions (see Figure 2). The V2V-PAEB Message usually goes to the Message Transmitter Model and then being sent out to the nearby vehicles. The safety decisions are sent to both the Actuator Models for triggering proper actions and the Display Model for displaying the simulation process and results. Figure 2 shows the architecture and information processing blocks of the V2V-PAEB model. Information processing is organized in four groups.
- The gray shaded block group describes the environment, vehicle and sensor setup for simulation.

- The non-shaded group is responsible for the on-vehicle sensor based pedestrian detection and V2V-PAEB message generation.

- The yellow shaded group is responsible for receiving V2V-PAEB messages and extracting the pedestrian information from messages.

- The blue shaded group is responsible for merging the pedestrian information from onboard sensors and received messages, and making car braking decisions.

Blocks for environment, vehicle and sensor setup (gray shaded blocks):

- Vehicle Kinematic Model block describes the locations and moving directions of the sensors on the vehicle, which need to be considered in calculating the collision distance and time.

- Sensor Model block describes the characteristics of sensors, such as range, field of view, error model, etc.

- Driving Scenario Model block describes the number of vehicles and objects involved in the simulation and their relative positions and motion trajectory.

- Vehicle Braking Model block describes the vehicle behavior for various braking commands.

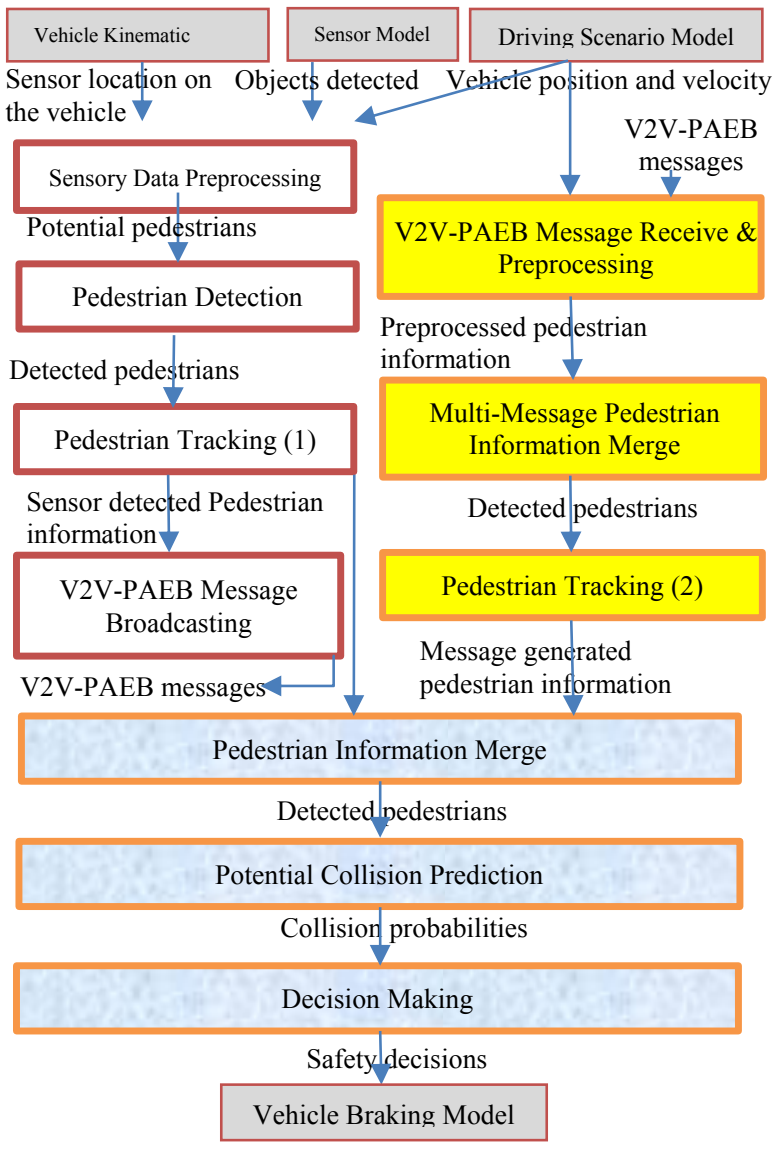

Figure 2. Information processing flow of V2V-PAEB simulation model. 
Blocks for vehicle sensor based pedestrian detection and $V 2 V$-PAEB message generation (non-shaded blocks):

Sensory Data Preprocessing block processes raw sensory data using simple cues and fast algorithms to identify potential pedestrian candidates. This stage needs to have high detection speed even at the expense of allowing false alarms.

Pedestrian Detection block applies more complex algorithms to the candidate object from the Sensory Data Preprocessing block in order to separate genuine pedestrians from false alarms.

Pedestrian Tracking (1) block tracks detected pedestrians overtime to get their trajectories.

V2V-PAEB Message Broadcasting block constructs and broadcasts V2V-PAEB Messages.

Blocks for V2V-PAEB messages receiving and pedestrian information extraction (yellow shaded blocks):

- V2V-PAEB Message Receive \& Preprocessing block periodically processes received messages to obtain the motion and state information of pedestrians contained in these messages.

- Multi-Message Pedestrian Information Merge block merges all the pedestrians extracted from different messages together to obtain a whole set of pedestrians that detected by other vehicles.

- Pedestrian Tracking (2) block tracks message extracted pedestrians overtime to get their trajectories.

Blocks for pedestrian information merging from onboard sensor and received messages, and making car braking decisions (blue shaded blocks):

- Pedestrian Information Merge block merges the two set of pedestrians information (one onboard sensor detected pedestrians and the other from received V2V-PAEB messages) together to obtain a complete set of detected pedestrians surrounding the host vehicle.

- Potential Collision Prediction block predicts the probability of collision between the host vehicle and pedestrians.

- Decision Making block generates warning to the driver if there is potential collision and generates braking commands if a collision is imminent [4].

The V2V-PAEB simulation model describes the information flow and processing for achieving pedestrian safety. To use it in simulation, it should be placed in a vehicle model. To reduce the complexity of developing this model, some third party simulation software (Such as PreScan, LabView and CarSim) can be used to provide complete or partial functions in some blocks. For example, PreScan software is used to provide blocks for environment, vehicle and sensor setup, and simulation display in this study. PreScan software also provided partial function support for message passing. Other blocks are developed either in Simulink or Matlab environment.

\section{Simulation Testing}

The proposed V2V-PAEB simulation architecture has been implemented and tested in PreScan environment [12]. PreScan provides several modules that the V2V-PAEB simulation needs. The intuitive graphical user interface (GUI) allows the construction of the experiment scenario and model the desired radar and camera sensors, while the Matlab/Simulink interface enables the integration of the V2V-PAEB simulation model. Following subsections describe the development and testing of the V2V-PAEB simulation using PreScan.

Figure 3 is a scenario chosen from paper [3] for testing the V2V-PAEB simulation architecture. In this scenario, five vehicles and one pedestrian are at an intersection. The traffic light changes from green to red when the pedestrian is still crossing the street. At the same time, vehicle 5 is approaching this intersection at a speed of $6.67 \mathrm{~m} / \mathrm{s}(24 \mathrm{~km} / \mathrm{h})$. The driver does not change the speed and keeps driving through this intersection. Both the pedestrian and vehicle 5 cannot see each other for their views are obscured by vehicle 2 . However, vehicle 1 and vehicle 2 can see this pedestrian. For this experiment scenario, two cases are run separately to check if the combined V2V-PAEB system works better than the PAEB only system. Combined V2V-PAEB system means information that detected by multiple vehicles will share their data in real-time through V2V network. The PAEB only system represents a scenario where vehicles detect objects individually without any communication.

Case 1: No vehicle has V2V capability and vehicle 5 is equipped with PAEB system. Since the sight of on-board sensors of vehicle 5 is blocked by vehicle 2, vehicle 5 cannot see the pedestrian until very late.

Case 2: Vehicles 1, 2 and 5 are equipped with V2V-PAEB systems. Vehicle 1 and 2 can detect the pedestrian early and broadcast this pedestrian information to vehicle 5 through V2V-PAEB messages.

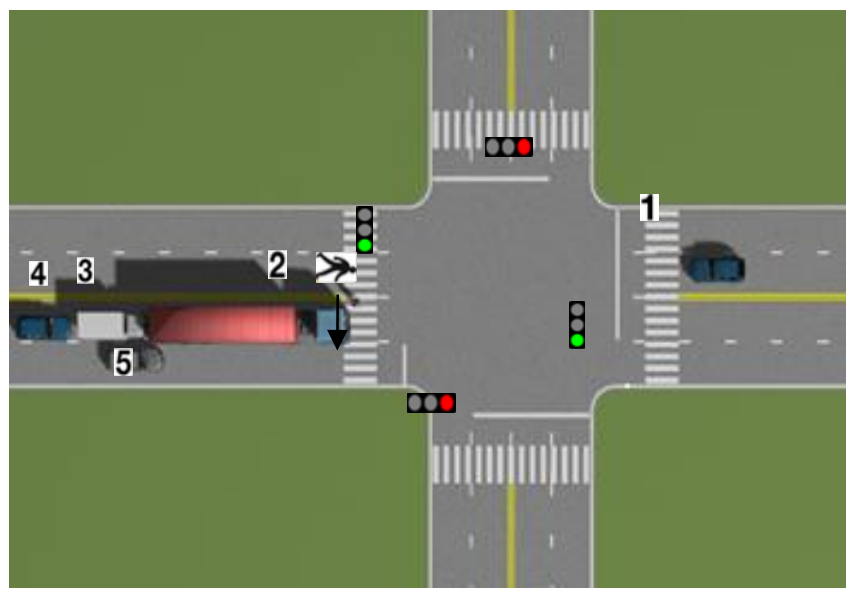

Figure 3. Simulation scenario used for testing the V2V-PAEB simulation model. 


\section{A. Build Experiment Scenario}

This experiment can be easily built in PreScan's GUI by using drag and drop actions to the library elements of road sections, infrastructure components (trees, buildings, traffic signs), actors (cars, trucks, bikes, and pedestrians), sensors (radar, camera, LiDAR, etc.), weather conditions (such as rain, snow, and fog) and light sources (such as the sun, headlights, and lampposts).

\section{B. Add the V2V-PAEB Model to the Vehicle Model}

The V2V-PAEB simulation model described above is inserted to the vehicle in the simulation. The Vehicle State Model, Radar Sensor Model, Camera Sensor Model and DSRC Receiver Model are connected to (or implemented in) the V2V-PAEB Simulation Model. The output of V2V-PAEB simulation model is connected to the DSRC transmitter model and actuator models.

\section{Simulation Result}

Two cases as described earlier are simulated. Table 4 compares the simulation results of case 1 and case 2. The simulation result of vehicle 5 for case 1 shows that there was a collision between vehicle 5 and the pedestrian with collision speed $0.67 \mathrm{~m} / \mathrm{s}$. The pedestrian was detected by the PAEB system when TTC was 0.39 seconds. Since it was too late and the PAEB system does not have enough time to react, the collision was not avoided but mitigated.

TABLE 4. THE COMPARISON OF THE SiMULATION RESUltS FOR CASE 1

\begin{tabular}{|l|c|c|} 
AND CASE 2 \\
\begin{tabular}{|l|c|c|}
\hline \multicolumn{1}{|c|}{ Items } & Case 1 & Case 2 \\
\hline Initial vehicle speed (m/s) & 6.7 & 6.7 \\
\hline Pedestrian detected time (s) (TTC) & 0.39 & 1.50 \\
\hline Full Braking generated time (s) (TTC) & 0.39 & 0.58 \\
\hline Collision Avoided [Yes/No] & No & Yes \\
\hline Collision Speed (m/s) & 0.19 & No collision \\
\hline
\end{tabular}
\end{tabular}

The simulation result of vehicle 5 for case 2 shows that the potential collision between vehicle 5 and the pedestrian was avoided successfully. The pedestrian was detected when TTC equaled to 1.50 seconds and the PAEB waited until $\mathrm{TTC}=0.58$ seconds to start full braking and the collision is avoided. It can be seen in TABLE 4 that the V2V-PAEB system on vehicle 5 in case 2 can detect pedestrians much earlier than that in case 1 so that the V2V-PAEB system can have more reaction time and has a better performance than PAEB system alone. If there is 0.4 seconds delay from generating the braking command to the actual start of mechanical braking due to communication and mechanical engagement, case 1 will have no mitigation and case 2 still avoid the collision. This is a demonstration that the V2V-PAEB system can compensate the limitations of the PAEB only system, and can improve pedestrian safety significantly.

\section{Other Simulation Cases}

Many scenarios have been identified that V2V-PAEB can be used to improve the pedestrian safety [3]. Figure 4 shows a 2-lane road example where pedestrian is crossing the road. The cars on the right lane are waiting to move, car 4 on the left lane moves fast. While the Pedestrian 7 is obscured by car 2, car 4 is about to crash pedestrian 7. In the simulation with V2V-PAEB support, car 4 can prevent the crash by getting pedestrian information from car 2 a few seconds earlier. Though whether this scenario has collision or not depends on the initial vehicle speed and TTC, however, TTC will be increased when V2V-PAEB is applied on all vehicles. Details of this simulation are not included in this paper, but the idea of the V2V-PAEB can be shown in Figure 4 clearly.

\section{DISCUSSION}

This study sets up the architecture of the V2V-PAEB system and establishes a simulation tool to support further study. Simple algorithms are implemented to demonstrate the operation of the V2V-PAEB system. Various issues of V2V-PAEB need to be further addressed. These issues include the effect of time delay in information transmission and processing to the performance of the system, the effect of sensor error to the performance of the system, and the communication jam caused by large amount messages sent by many vehicles.

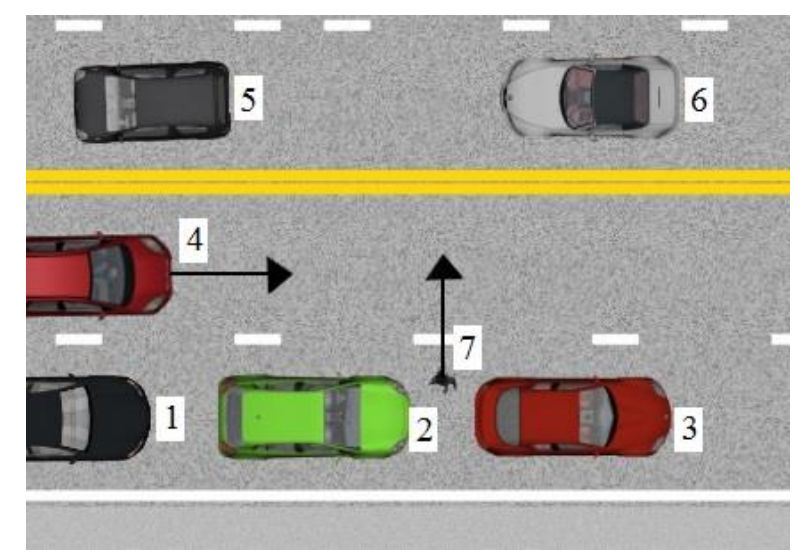

Figure 4. Simulation of V2V-PAEB - pedestrian dart out.

\section{CONCLUSION}

This study described the idea of using the V2V-PAEB system to compensate the limitations of PAEB system that has short detection range and unable to detect obscured objects. The approach integrates the capabilities of V2V and PAEB to allow the information of pedestrians detected by PAEB system to be shared in the V2V network. Theoretically, the V2V-PAEB system should have better performance than PAEB system because other vehicles can report the pedestrians that one vehicle's PAEB system failed to detect. Architecture for V2V-PAEB system is described. It decomposes various tasks associated to the V2V-PAEB system into smaller specific problems. The input and output parameters of the V2V-PAEB system as well as its each block are also defined. The simulation has shown that the proposed V2V-PAEB has definite advantage over the PAEB alone system. The simulation provides a good foundation and tool for further V2V-PAEB study.

\section{ACKNOWLEDGEMENT}

This project was funded by the US Department of Transportation, Crash Imminent Safety (CrIS) University 
Transportation Center (UTC). The authors also thank TASS International generous support for providing the PreScan simulation tool.

\section{REFERENCES}

[1] J. Harding, G. Powell, R. Yoon, J. Fikentscher, C. Doyle, D. Sade, M. Lukuc, J. Simons, and J. Wang, "Vehicle-to-Vehicle communications: Readiness of $\mathrm{V} 2 \mathrm{~V}$ technology for application," Washington, DC: National Highway Traffic Safety Administration. DOT HS 812 014, Sep 16 2014, http://www.nhtsa.gov/stat...for-Application-812014.pdf

[2] E. Coelingh, A. Eidehall and M. Bengtsson, "Collision Warning with Full Auto Brake and Pedestrian Detection - a practical example of Automatic Emergency Braking," $201013^{\text {th }}$ International conference on Intelligent Transportation Systems (ITSC), Sept 19-22, 2010, pp. 155-160.

[3] M. Liu, S. Chien, Y. Chen, "Improve Road Safety Using Combined V2V and Pre-Collision Systems," The 24th International Technical Conference on the Enhanced Safety of Vehicles (ESV2015), Gothenburg, Sweden on June 8-11, 2015. Paper Number 15-0431.

[4] T. Gandhi, M. Trivedi, "Pedestrian Protection Systems: Issues, Survey, and Challenges," IEEE Transactions on Intelligent Transportation Systems, Sept. 2007, pp. 413-430.

[5] Bartsch, A., F. Fitzek, and R. H. Rasshofer. "Pedestrian recognition using automotive radar sensors." Advances in Radio Science 10.4 (2012): 45-55.

[6] A. Bhawiyuga, H. Nguyen. H. Jeong, "An Accurate Vehicle Positioning Algorithm Based on Vehicle-to-Vehicle (V2V) Communications," 2012 IEEE International Conference on Vehicular Electronics and Safety (ICVES), 24-27 July. 2012.

[7] L. Bowen, Y. Danya. "Calculation of Vehicle Real-time Position Overcoming the GPS Positioning Latency with MEMS INS," IEEE International Conference on Service Operations, Logistics, and Informatics (SOLI), 8-10 Oct. 2014.

[8] B. Tang, S. Chien, and Y. Chen, "Obtain a Simulation Model of a Pedestrian Collision Imminent Braking System Based on the Vehicle Testing Data," 17th IEEE Int.Conf. on Intelligent Transportation Systems, Oct 8-11, 2014, Qingdao, China.

[9] O. Trullols-Cruces, M. Fiore, J.M. Barcelo-Ordinas, "Understanding, modeling and taming mobile malware epidemics in a largescale vehicular network," World of Wireless, 2013 IEEE 14th International Symposium and Workshops on Mobile and Multimedia Networks (WoWMoM), June 4-7, 2013.

[10] Y. Chen, K. Shen, S. Wang, "Forward Collision Warning System Considering Both Time-to-Collision and Safety Braking Distance, 2013 8th IEEE Conference on Industrial Electronics and Applications (ICIEA). June 19-21, 2013.

[11] M. Heesen, M. Dziennus, T. Hesse, A. Schieben, "Interaction design of automatic steering for collision avoidance: challenges and potentials of driver decoupling," Intelligent Transport Systems, IET. 22 Jan. 2015.

[12] M. Tideman, M. Noort, "A Simulation Tool Suite for Developing Connected Vehicle Systems," 2013 IEEE Intelligent Vehicles Symposium (IV), June 23-26, 2013. 